\title{
Significados simbólicos de los pacientes con enfermedades crónicas*
}

\author{
SIGNIFICADOS SIMBÓLICOS DOS PACIENTES COM DOENÇAS CRÔNICAS
}

SYMBOLIC MEANINGS OF THE PATIENTS WITH CHRONIC DISEASES

Luz Angélica Muñoz ${ }^{1}$, Yocelyn Price ${ }^{2}$, Liliana Gambini ${ }^{3}$, Maguida Costa Stefanelli ${ }^{4}$

\section{RESUMEN}

La investigación presenta tres universos culturales que sufren Hipertensión Arterial, Diabetes y Artrosis, describiendo las distintas dimensiones y la interpretación de los significados. Se utilizó el método etnográfico, utilizando la entrevista etnográfica y la observación participante y, en la interpretación, el interaccionismo simbólico y otros autores. Los aspectos éticos de la investigación con seres humanos fueron respetados. Surgen convergencias en las dimensiones en que son percibidas las tres enfermedades: la pérdida del bienestar y soporte social, miedo a la invalidez y muerte, percibiendo la familia y aceptando la cronicidady, valorizando la medicina alternativa. El cuidado de enfermería es percibido por esta clientela con ambigüedad.

\section{PALABRAS CLAVE}

Enfermería.

Anciano.

Antropologia cultural.

\author{
ABSTRACT \\ The investigation presents \\ three cultural universes of \\ those suffering from arterial \\ hypertension, diabetes and \\ arthrosis, describing the \\ different dimensions and \\ interpreting their meaning. \\ The ethnographic method was \\ utilized, using the \\ ethnographic interview and \\ participant observation and \\ for the interpretation, the \\ symbolic interactionism and \\ other authors. Convergences \\ in the dimensions arise in \\ which the three diseases are \\ perceived: the loss of well- \\ being and social support, fear \\ of disability and death, \\ perception of the family and \\ accepting the chronicity, \\ interest in alternative \\ medicine. The informants of \\ this study perceive nursing \\ care with ambiguity.
}

\section{KEYWORDS}

Nursing.

Aged.

Antropology, cultural.

\section{RESUMO}

A investigação apresenta três universos culturais de portadores de Hipertensão Arterial, Diabetes e Artrose, descrevendo as distintas dimensões e a interpretação dos significados, utilizando o método etnográfico, o Interacionismo Simbólico e diferentes autores. Os aspectos éticos da pesquisa com seres humanos foram respeitados. Surgem convergências nas dimensões em que foram percebidas as três doenças: a perda do bemestar e apoio social, medo da invalidez e morte, percebendo a familia e aceitando a cronicidade e valorizando a medicina alternativa. $O$ cuidado de enfermagem é percebido pelos informantes do estudo com ambigüidade.

\author{
* Project I GAVE 74-00 \\ and Project I GAVE 35-02 \\ 1 Profesor Titular. Doctor \\ en Enfermería, \\ Directora Escuela de \\ Enfermería, \\ Universidad Andrés \\ Bello, Santiago, Chile. \\ Imunoz@unab.cl \\ 2 Licenciado en \\ Enfermería, Secretaria \\ Académica Escuela de \\ Enfermería, \\ Universidad Andrés \\ Bello, Santiago, Chile. \\ 3 Médica Fisiatra, \\ Directora Centro de la \\ Discapacidad, \\ Universidad Andrés \\ Bello. \\ 4 Professora Titular de \\ Enfermería de la Escola \\ de Enfermagem da \\ Universidade de São \\ Paulo. Asesora Consejo \\ Director Instituto de \\ Psiquiatría do Hospital \\ das Clinicas da \\ Faculdade de Medicina \\ da Universidade de São \\ Paulo. \\ mcstefanelli@terra.com.br
}

\section{PALAVRAS-CHAVE}

Enfermagem.

Idoso.

Antropologia cultural. 
Luz Angélica Muñoz

Yocelyn Price

Liliana Gambin

Maguida Costa Stefanell

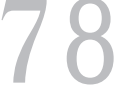

Rev Esc Enferm USP 2003; 37(4): 77-84.

\section{INTRODUCCIÓN}

En Chile las enfermedades crónicas desde hace décadas ocupan los primeros lugares de morbilidad y a la vez sus complicaciones constituyen las principales causas de discapacidad en el adulto mayor.

El adulto mayor se ve afectado por variadas enfermedades que destacan las encuestas de morbilidad de Medina, Kaempffer ${ }^{(1)}$ realizadas en Santiago. Señalan que el $25 \%$ de las familias tiene algún miembro que padece de una enfermedad aguda, 3,1\% con algún miembro que ha sufrido un traumatismo y el $39,5 \%$ tiene alguno con enfermedad crónica. De este modo, las enfermedades crónicas más percibidas son: la hipertensión arterial, los problemas de columna y artropatía, diabetes, bronquitis crónica, neurosis entre otras. Esto sería producto de una mayor percepción de las enfermedades crónicas que han generado un aumento de las prestaciones, consultas, visitas médicas. Es así, como este grupo padece de enfermedades las que aumentan a partir de los 70 años. En relación a la invalidez, la principal causa de ella son las afecciones del aparato locomotor.

En Chile, las enfermedades del aparato circulatorio, entre ellas la hipertensión arterial, son la tercera causa de muerte en adultos mayores constituyendo un 3,4\%. En 1991 fue la primera causa de consulta a nivel primario (9,3\%), lo que hacen que sea la cuarta prioridad de salud del país ${ }^{(1)}$.

En Chile, el 3,2\% de la población dice haber sido diagnosticado con Diabetes, porcentaje equivalente a más de 300.000 personas, según la Encuesta de Calidad de Vida y Salud MINSAL-INE, en tanto se estima que una cifra similar padece la enfermedad pero no ha sido diagnosticada y por lo tanto, enfrenta un mayor riesgo de presentar complicaciones ${ }^{(2)}$.

La diabetes representa la séptima causa de muerte en Chile, según datos del Ministerio de Salud de 1998, principalmente por la enfermedad cardiovascular asociada a la misma. Es, asimismo, la principal causa de amputación de origen no traumático y también una de las principales responsables de la ceguera e insuficiencia renal crónica ${ }^{(2)}$. En cuanto a las enfermedades osteoarticulares en sus diversos diagnósticos, presentaron 326 muertes, ségun el Anuario de Demografía, en cuanto a la morbilidad no existen datos oficiales del Ministerio de Salud, sin embargo hay servicios que tienen su propio banco de datos, habiéndose encontrado que la artrosis en el Hospital Base Valdivia, presenta la mayor prevalencia alcanzando un $70 \%$ en adultos mayores de 70 años ${ }^{(2-3)}$.

$\mathrm{La}$ artrosis es la primera causa de consulta reumatológica que representa, también, los años potencialmente perdidos (AVPP). Los pacientes reportan que tienen una calidad de vida muy pobre si se comparan con otras que no tienen esta enfermedad ${ }^{(4)}$.

Las molestias crónicas traen limitaciones que muchas veces no son aceptados por los portadores de ellas por falta de conocimiento de cómo enfrentarlas.

Las enfermedades crónicas desde hace décadas ocupan los primeros lugares en morbilidad y mortalidad de adultos. En nuestras investigaciones hemos seleccionado, la hipertensión arterial, diabetes mellitus y enfermedades osteoarticulares como la artritis y artrosis por ser enferme-dades prioritarias del Adulto Mayor, que requieren cada vez más de cuidados responsables de la salud y al mismo tiempo de un cuidado sensible por parte del equipo de salud y la familia.

\section{DESARROLLO}

El objetivo de este estudio es describir la vivencia y las convergencias de las dimensiones estudiadas en tres universos culturales de la clientela que ha sufrido Diabetes Mellitus(14), Hipertensión Arterial(16) y Enfermedades OsteoarticularesArtrosis (20). Los informantes fueron adultos mayores (entre 60 y 65 anõs), de ambos sexos, beneficiários del Sistema de Salud Público y consultorios de atención primaria y especialidades.

Se adoptó el método etnográfico y el Interaccionismo simbólico para describir e interpretar los significados del proceso salud enfermedad que vivencian los tres universos culturales indicados ${ }^{(5-6)}$.

Utilizamos la observación participante y la entrevista etnográfica de Spradley ${ }^{(5-6)}$. Los datos fueron registrados para su 
sistematización en dominios culturales, taxonomías y análisis de temas. Las entrevistas en profundidad se iniciaron con una pregunta inicial abierta "¿Cómo ha sido para usted esta enfermedad?", seguida de un grupo de preguntas descriptivas, estructurales y de contraste acerca de la vivencia y relacionadas a la respuesta de la pregunta inicial ${ }^{(7)}$.

Finalmente, se hizo un análisis reflexivo para buscar los significados culturales de estos procesos que vivencian los adultos mayores y la posterior validación de los datos.

Se consideraron los principios filosóficos y éticos en la investigación, los datos fueron obtenidos con la aceptación y autorización de los participantes. La garantía del anonimato de los informantes es una forma de protección de sus derechos. Las entrevistas fueron grabadas con consentimiento. Los informantes fueron adultos mayores, con diagnóstico de Hipertensión Arterial y Diabetes Mellitus y Enfermedades osteoarticulares.

\section{Pérdida de bienestar y soporte social}

La expresión con que la clientela describe la hipertensión es: "una ruina total". Esta dimensión constituye una dimensión de pérdida del bienestar que interfiere en varios aspectos de sus vidas, tales como la destrucción de la red social de apoyo.

El contenido de sus discursos expresan:

Me encuentro un hombre inútil

Una carga para toda la vida

Ahí cambian las cosas, las amistades, casi todo. Limitado confinado a la casa.

Nuestros pacientes le otorgan a la hipertensión una fuerte connotación de pérdidas afectivas y sociales, señalando la influencia de estas relaciones en todos los ámbitos de su vida.

En la diabetes mellitus el universo cultural la describe como:

es una enfermedad peligrosa, muy difícil, que no sana, hasta desexualiza, (...) es igual que el cáncer, nadie la ataja, (...) es una enfermedad interesante, ellos no saben tratar la diabetes,

de acuerdo a su visión de mundo y a su experiencia, debido a las complicaciones que produce en diversas partes del cuerpo dejando a la persona incapacitada o inválida para desempeñar su trabajo ${ }^{(8)}$.

En otro universo cultural, los pacientes nombran a la Diabetes como: "Esa cosa..esa maldita enfermedad, enfermedad del diablo" y debido a que no la pueden controlar, expresan:

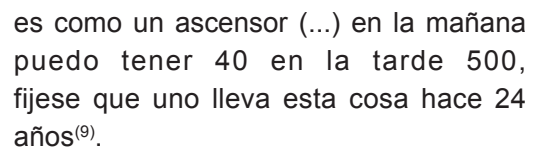

Como se puede evidenciar, los universos estudiados nombran la enfermedad con símbolo o metáfora.

Estos discursos describen a la diabetes como una enfermedad que irrita, invade, descontrola por lo que la vida del yo sufre cambios por los cuales nunca más será el mismo yo sano de antes y pasará a vivir en el mundo de la enfermedad con restricciones y control.

Se presenta entonces como una enfermedad no comprendida, en un momento en que el supuesto de la medicina oficial es que todas las enfermedades estén prácticamente controladas o curadas.

La expectativa, al no tener salud, es ser sano y los discursos de la clientela evidencian urgencia por tener salud. Sienten que no tienen un futuro provisorio, el pasado es confuso y por lo tanto no hay nada que pueda ser útil al yo.

En las enfermedad es osteoarticulares, los pacientes perciben la enfermedad por los síntomas, especialmente el dolor y la dificultad para movilizarse. La caracterizan con la expresión: "Es una enfermedad que se siente", por lo tanto una enfermedad propia. Este universo cultural tiene muy buena información acerca de los servicios de salud y su referencia cuando presentan dolor. El dolor gatilla la consulta. El dolor permea los discursos de este grupo de pacientes y lo expresan como "me falló la rodilla, no puedo salir, no puedo caminar, yo salía mucho"(10)

\section{Miedo de la invalidez y muerte}

En la hipertensión surge en estos universos, el miedo a las complicaciones de la enfermedad que les producen invalidez,
Significados simbólicos de los pacientes con enfermedades crónicas 
Luz Angélica Muñoz

Yocelyn Price

Liliana Gambini

Maguida Costa Stefanelli asociado al miedo a la dependencia de otras personas se complementa con el miedo a la muerte por el riesgo de enfermedad cerebrovascular.

Todo ello, genera angustia y sufrimiento por los cambios que deben hacer para modificar hábitos que afecta en sus relaciones. Esto es expresado como

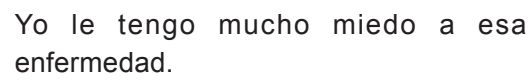

En la diabetes el universo cultural expresa su sentimiento a través del miedo, sufrimiento desesperanza y dolor, expresan

\section{Cuando acabe este sufrimiento, este dolor, visitaré a mi familia.}

Sólo quiero pegarme un tiro, ya llevo todos estos dedos (3) amputados, estoy muy deprimido, ya no doy más, es morir día a día, perdiendo a pedazos el cuerpo...

Sufro mucho con mis piernas, es terrible no andar en sus pies (...)cuando iba a pensar que iba estar en silla de ruedas (...), nunca me explicaron habría ido al control si hubiera sabido que iba a perder mis piernas.

Se percibe en los universos culturales una percepción peculiar de la enfermedad incurable. La diabetes es entendida como debiendo ser una preocupación del otro, porque los profesionales los someten a una serie de obligaciones de índole preventiva como control para mantener adhesividad al programa, dejar de fumar, no comer dulces no engordar y otros que la mayoría están preparados para practicar. Esa necesidad es fuertemente sentida en el momento que las complicaciones surgen.

Para gran parte de los pacientes la diabetes se perfila como amenaza al futuro trayendo inseguridad, angustia.

Con la diabetes me siento limitada por las restricciones que normalmente le imponen al diabético (...) me preocupo por las complicaciones, es una enfermedad incurable llega a ser hasta fatal (...) la muerte llega un día y no hay manera de luchar.

Las personas sienten que el futuro imaginado no será posible porque su fin llegará y que con la enfermedad no será posible modificar su estilo de vida. Así, la diabetes se presenta como una enfermedad cargada de fatalidad como enfrentándolos con la idea de muerte y miedo a lo desconocido que los afectan de diversos modos. Vivencian la desesperanza y la fatalidad con amenaza continua de finitud.

Otro tipo de miedo, es la pérdida de partes del cuerpo por amputaciones. Todos los discursos de pacientes que han tenido amputaciones únicas o múltiples expresan esto en el fenómeno del miembro fantasma.

Señalan el proceso de reconocimiento de pérdidas de segmentos de su cuerpo, sintiendo estas pérdidas como parte de su imagen corporal.

El dolor del miembro amputado, terminará cuando el paciente sea capaz de utilizar la muleta y sentir el ruido considerándola como parte de su cuerpo expresivo ${ }^{(12)}$.

En las enfermedades osteoarticulares las vivencias del universo con esta enfermedad, se inicia mayoritariamente con dolor físico y dolor espiritual, este último es mayor que el dolor físico ya que temen que no les crean en su dolencia y que no comprendan el significado de la enfermedad.

En sus discursos señalan el cambio de su cuerpo y el cambio de las funciones. Refieren que "antes era super activo" y como la enfermedad comienza poco a poco evidenciando cambios físicos que no son notorios, por lo que la familia no comprende el inicio de la enfermedad y a medida que transcurre el tiempo, al disminuir sus capacidades físicas, no pueden cooperar con quehaceres del hogar. Como resultado de la enfermedad sienten que

se me enchuecaron los dedos y me afectó en una cosa tan simple como no poder pegar un botón, no poder caminar .

a mi, se me caían los platos, no podía pararme ni sentarme, por lo tanto, no podía hacer nada.

Sienten que la enfermedad trae dolor agudo, día y noche, siendo una enfermedad que no se nota, sino que se siente, lo que les ocasiona sentimientos de ira consigo mismo, por no realizar actividades que realizaban cuando estaban sanos.

Los pacientes se sienten derrotados por un sentimiento de inutilidad, anulación e impotencia. 
Estas expresiones consideradas en términos simbólicos serían una señal o una indicación de la existencia de que algo está mal en su organismo.

De este modo, esta clientela, ante eventos estresantes como complicaciones de la enfermedad se sienten ansiosos y amenazados, surgiendo emociones de rabia, incertidumbre que conlleva al surgimiento del miedo. Nuestros pacientes sufren de temor a perder su autonomía, a la soledad, a las consecuencias de la enfermedad, a las limitaciones físicas. No desean perder su independencia y sienten incertidumbre en relación a las características del cuidador que asumiría su cuidado, tomando decisiones por ellos, y finalmente miedo a la incapacidad por la pérdida de la red social.

Por lo tanto, el miedo siempre constituye una respuesta a una causa conocida, presintiendo los riesgos potenciales de una situación; se trata en estos casos del miedo anticipatorio en la que los pacientes se imaginan el riesgo derivado de la suposición que volverá a suceder lo ya ocurrido, como recuerdos penosos de su última hospitalización, muerte de un ser querido, entre otros.

\section{Valoración de medicina alternativa}

La clientela hipertensa valora las plantas medicinales, realizando acciones de autocuidado para bajar la presión. Por otra parte, los clientes refieren distintas formas de adquirir estos conocimientos, diciendo "una viejita me dijo..." otros dicen "la vecina, las viejitas me dijeron.", "me lo dijo una turista" otros mencionan que incluso ellos mismos transmiten este conocimiento, "yo les doy esta receta", en la mayoría de los casos este saber popular se encuentra en poder de personas añosas, quienes por esta situación tienen un conocimiento dado por la experiencia adquirida a través de los años y transmitida de generación en generación. A medida que ellos obtienen resultados positivos en la práctica de estos conocimientos, los transmiten a otras personas que padecen de la misma enfermedad.

Así, junto con las razones y los efectos de cumplir el tratamiento, podemos encontrar en los discursos de nuestros pacientes hipertensos, elementos que son usados por ellos para bajar la presión arterial, principalmente el uso de plantas medicinales tales como: ruda, natre, toronjil, matico, triaca y zarzaparrilla, expresando :

El natre es el que no deja subir la presión....les tengo fe a estos remedios, porque yo de la edad que tengo primera vez que piso hospital y veo doctores.

De este modo los pacientes señalan cómo obtienen las plantas que necesitan como parte de su cuidado, cómo las preparan para su consumo y la frecuencia con que las toman, podemos ver también el efecto que tienen en ellos. Nuestros pacientes utilizan estas medidas, sin excluir las indicaciones médicas. Otros hacen referencia al inicio del uso de estas plantas y a la forma de tomarlas, diciendo

las probé y tienen buen gusto, se toman en vez del te (...)me ha hecho bien, (.. )ahora estoy tomando de esa agüita, junto con los remedios que me dan.

Con esto queda explícita la intención de utilizar estas medidas como complemento del tratamiento médico tradicional, y que cada planta tiene su forma de preparación y dosis. De igual modo relatan

cuando noto que tengo la presión alta a las doce tomo un remedio de ruda, un tecito de ruda (...). hay que tomarla promediadamente

expresando claramente la dosis en que hay que tomar la planta, y el momento cuando la toma

yo le voy a contar algo que al médico no le he dicho, un día me sentía mal, una viejita me dijo que tomara agüita de natre, y eso me baja la presión (...) se pone un poquito de natre seco y se pone a hervir, hace bien, dura harto (...) como tres meses el efecto.

Existe pues, un conocimiento que es propio de los pacientes y que no comparten con los profesionales de salud, percibiendo, además, que tampoco éstos demuestran interés por este conocimiento.

También los clientes relatan "cuando me siento mal del corazón yo tomo mis remedios y si no me pasa tomo agüita de toronjil". Esto denota que la hierba toma sólo cuando se siente mal, cabe destacar que describe claramente una secuencia del uso de sus remedios, utilizando primeramente los medicamentos de la medicina tradicional, y luego frente al fracaso de estos utiliza los remedios naturales.
Significados simbólicos de los pacientes con enfermedades crónicas 
Luz Angélica Muñoz

Yocelyn Price

Liliana Gambini

Maguida Costa Stefanelli
En la diabetes mellitus, el universo cultural usaba también las plantas específicas, conocidas por su eficacia, a través de las interacciones y de intercambio entre los propios pacientes. Las hierbas fueron usadas en forma de té e infusiones, a veces utilizan una mezcla de diferentes tipos de plantas. Para esa preparación relataron los pasos que siguen tales como una receta de cocina, cuantificando cada una de ellas y cuando era necesario sustituyendo aquellas que eran similares.

La clientela que sufre enfermedades osteoarticulares, utilizan el sistema tradicional de salud con medicamentos, kinesiterapia, que incluye diversos tratamientos y un sistema de medicina alternativa, en los que destacan, acupuntura, homeopatía, dieta y aura.

Los tratamientos de la medicina oficial nombrados por estos pacientes son: ultratermia, ultrasonido, láser, ejercicios terapéuticos,bicicleta-ergométrica, masoterapia, hidroterapia, termoterapia superficial. Complementan sus tratamientos con acupuntura, plantas medicinales, como la zarzaparrilla. Otros tratamientos señalados fueron: hongos del tibet, homeopatía, cataplasma de barro, dietas, sanación cuántica y estudio del aura. El grupo percibe la eficacia de los tratamientos alternativos y desconfían de los tratamientos de la medicina oficial.

\section{Expresan:}

El médico que estudió acupuntura. (...) me
hizo 3 aplicaciones.
!nunca tuve un dolor!
Yo había utilizado antes la homeopatía y
me hizo bastante bien (...) siempre que no
sea antagónico al tratamiento médico
(...) la sanación cuántica (...) eso es la
relajación a través de la imposición de
manos, tratando de movilizar energías, (...)
me siento bien con este tratamiento(...) mi
pensamiento era que la prótesis estaba
fallando (...) Yo tuve una mala enseñanza,
el uso permanente del bastón (...) estuve
6 años en que no solté el bastón y ello
afecta la columna (...) si usted me ve va a
ver la $S$ que tengo en la columna (...)le
tengo miedo a los antiinflamatorios,
porque uno sabe que tiene que morirse,
pero quiere morirse decentemente!. Sin
tanta cosa! Esa es la verdad!.

\section{Percibiendo a la Familia}

Las percepciones de los tres universos culturales, si bien la familia se considera un valor, hay ambivalencia en sus relaciones con ella.

Para un grupo existe disarmonía en la dinámica familiar, que ocasiona conflictos por la responsabilidad que ejerce el miembro de la familia que cuida por la presión que ejerce de las restricciones alimentarias del paciente. "Me molesta, me pasan a llevar porque sufro y dependo de mi familia"

Otros grupos, si bien aceptan las recomendaciones del cuidador, perciben apoyo y cariño "Se preocupaban por mi", "la gente me quiere y más mi familia, sé que nunca me abandonarán".

La familia es percibida con ambigüedad, ya que se le considera un grupo controlador que al igual que los servicios, los califican como transgresores de las reglas. También la perciben con un rol fundamental de apoyo para adherir al tratamiento y luchar con un sistema normativo.

Hay un grupo de clientes que tienen comportamientos que lo alejan de lo que los servicios consideran un buen paciente, o que un familiar enfermo debería tener.

Algunos se revelan contra las reglas o cualquier forma de control, tienen sentimientos de privación, desesperanza, lo que interfiere en la comunicación con los otros.

Douglas, relaciona el tema con la polución social, o sea el peligro, así el cliente puede ser una amenaza cuando no sigue las orientaciones, constituyéndose en un peligro social que amenaza el sistema ${ }^{(10)}$.

Las creencias que existen en la clientela que presenta enfermedades osteoarticulares condicionan sus comportamientos respecto a su salud, e influencian la dinámica familiar:

\footnotetext{
en un principio no me entendían y para uno es terrible y duele más que el dolor físico, a pesar que el dolor físico es tremendo, pero el hecho de que no le crean a uno es peor i (...) bien me ayudaban mucho, si iba a salir, al principio yo andaba sola no me afectaba nada, para entrarme al baño tiene que entrarme una persona por que... tengo que entrar bien, por temor a caerme.
} 
También a los pacientes no les agrada demostrar su intenso sufrimiento, a la familia, expresando su sentir de esta forma:

(...) ni a mi familia ni a nadie, mis dolores son míos ¡¡no!! Yo no lo demuestro, me pillan no mas por que a veces ando muy como la rastra, pero ¡no! No! A nadie a nadie, ni a mi hijo,ni a mi hermana, a nadie ¡es solo mío! No me gusta que me compadezcan, yo creo que es por eso, creo que es por eso.

Sin embargo, para muchos pacientes, el apoyo familiar es un pilar fundamental en el inicio y transcurso de su enfermedad, ya que cuando su familia le entrega amor y comprensión, los ayuda a que crezca esa fuerza interior para seguir adelante con sus vidas. Los discursos expresan:: "me cuidan harto", "es muy importante el apoyo que da la familia", este apoyo que reciben también les ayuda a no sentirse inútiles o que son una carga para el resto, y así sienten que todavía les quedan muchas cosas por hacer, y que no pueden darse por vencidos.

Otros lo expresan diciendo:

\begin{abstract}
Mira mi papá ya hace dos años que me falta, pero él siempre me apoyó bastante . El siempre fue súper preocupado de mí, mi mamá... que ellos no se preocupen en este aspecto, ella ahora tiene ochenta y cinco años, entonces yo, ya ahora no le digo esas cosas. mi hija, ella es bien considerada. Mis hermanos, con ellos siempre nos hemos mantenido unidos (...).

A parte que soy la única mujer y me cuidan harto (...) paso acompañada con mi mamá y bueno, con mis hermanos que igual no viven conmigo, pero es como si vivieran. Viven pendientes de la casa (...) y es mejor. Es muy importante el apoyo de la familia, y no solo de la familia, sino que también, bueno, que son también de la familia pero mis primos que son como hermanos, o sea, en mí me pasa algo y todos corren a ver qué me pasa.
\end{abstract}

Se reconoce el rol de la familia en el cuidado de las personas que sufren las patologías mencionadas, así la salud y la calidad de vida de un integrante enfermo influye en el cuidado brindado por la familia. El rol de la familia implica el cariño, y la aceptación de la persona enferma. Las mujeres son quienes proveen el cuidado en la familia y quienes en nuestro medio responden a una expectativa social y cultural.

\section{Aceptando la enfermedad crónica}

Percibimos que esta clientela acepta la cronicidad de la enfermedad con sus consecuencias de destrucción y pérdida del valor de la salud, sin embargo intentan tener energía y fortaleza y luchar para vencer la enfermedad, recuperando su bienestar en función de sus vínculos y roles que desempeñan en su comunidad.

Para esta clientela la cronicidad se refleja también en el dolor crónico, específicamente en las enfermedades osteoarticulares y en aquellos que han sufrido la amputación de uno de sus miembros, lo que se transforma en la enfermedad misma, perdiendo su condición de alerta. Esto produce alteraciones en la afectividad y comunicación en la relación con los otros:

no soy $100 \%$ el mismo de antes (...), uno pasa el día con dolor, a uno le cambia el ánimo, es todo el día, día y noche con dolor (...)es de por vida (...) Uno le va ganando a la enfermedad, aunque sea una carga para toda la vida.

El cuidado brindado por la enfermera en nuestros escenarios culturales representa un apoyo que la clientela valora para su bienestar con sus sentimientos, aprecian su escuchar atento y delicadeza en su relacionamiento. Una dimensión que se muestra en los pacientes con diabetes es la relación empática con el equipo con el que interactúa, destacando en forma especial a las enfermeras y los significados de relación pacienteenfermera en el ámbito verbal y no verbal. En la mayoría de los discursos es evidenciada la visibilidad de las enfermeras, por su actitud humana y expresiva. La fe y confianza en los cuidados, en las actividades que hacen con el paciente, en sus características y en los sentimientos que ellos proyectan otorga a esta clientela sentido a su existencia. En el universo de pacientes hipertensos, no se percibe la visibilidad descrita anteriormente, por lo que creemos que el equipo de salud, y especialmente la enfermera deben favorecer un relacionamiento con comunicación empática y abandonar algunas de sus actitudes y creencias, para fortalecer el cuidado que ella brinda a nivel de los programas de salud.
Significados simbólicos de los pacientes con enfermedades crónicas 
Luz Angélica Muñoz

Yocelyn Price

Liliana Gambin

Maguida Costa Stefanell

\section{CONSIDERACIONES FINALES}

Los adultos mayores que sufren este tipo de enfermedades crónicas, convergen en las dimensiones de: pérdida del bienestar y soporte social, miedo a la invalidez y a la muerte, en las formas como perciben la familia y en la aceptación de la cronicidad.

Se hace necesario redimensionar los espacios que enfermería tiene y puede tener en los programas del Adulto mayor para un cuidado sensible y coherente con la clientela, atendiendo a la cosmovisión de los pacientes y de la familia que beneficiará la adhesividad al programa y el bienestar de los pacientes.

\section{REFERENCIAS}

(1) Medina Lois E, Kaempffer AM. Mortalidad del adulto en Chile. Rev Med Chile 2000; 128: 1144-49.

(2) Ministerio de Salud. Departamento de Epidemiologia. Situación de salud Chile. Santiago; 2000 .

(3) Hospital Base Valdivia. Estadística Policlínico de Reumatología. Valdívia; 1999. (mimeografado)

(4) Fauci AS. Word health Organization morbmort: Weekly Report 2000; 49:366

(5) Spradley J. The ethnographic interview. New York: Holt Rinehart \& Winston; 1979.

(6) Spradley J. Participant observation. New York: Holt Rinehart \& Winston; 1980.

(7) Muñoz LA, Stefanelli MC, Calvo MJ, Jerez V. Fundamentos de la enfermería transcultural. Rev Enferm Invest Desarro 1997; 1:5-11.

(8) Muñoz LA. Vivenciando a diabete: um estudo etnográfico. Rev Esc Enferm USP 1995; 29: 91-103.
La relación dialógica y la interacción recíproca que se desarrolla en la relación enfermera-cliente, es fundamental ya que el cuidado se dirige a apoyar a estos pacientes en la prevención, recuperación y rehabilitación de la salud, satisfaciendo, sus necesidades humanas fundamentales.

La familia permea las dimensiones convergentes con su papel fundamental de apoyo para el seguimento del tratamiento, cuidado y aceptación de la enfermedad. Por lo tanto podemos afirmar como Stefanelli ${ }^{(13)}$ que, en el editorial de una revista sobre la familia, refiere "la familia está presente, en todas las circunstancias de nuestras vidas, sean circunstancias adversas o no."

(9) Muñoz LA, Jerez V, Calvo MJ. Vivenciando la enfermedad y el cuidado del ser diabético. Cienc Enferm 1998; 4:89-98.

(10) Muñoz LA, Price Y, Maureira P, Gambini L. Información y utilización de los servicios clínicos de adultos con enfermedades osteoarticulares: un análisis etnográfico. [Presentado en la XVII Conferencia Mundial de Promoción de la Salud y Educación para la Salud; 200115 al 20 de julio, Paris].

(11) Price Y, Arellano P, García M, Burgos D, Peñailillo J, Muñoz LA. Vivenciando la Hipertensión Arterial en consultorios de atención primaria. Fam Saúde Desenv 2000; 2:65-70.

(12) Martins J. Um enfoque fenomenológico do currículo: educação como poiésis. São Paulo: Cortez, 1992.

(13) Stefanelli MC. Familia.. Familia..., apesar da adversidade. /editorial/ Fam Saúde Desenv $2001 ; 3$. 